QMUL-PH-02-18

hep-th/0209115

revised March 2003

\title{
Four-dimensional topological Einstein-Maxwell gravity
}

\author{
P. De Medeiros and B. Spence \\ Physics Department, \\ Queen Mary, University of London, \\ Mile End Road, London E1 4NS, UK
}

In memory of Sonia Stanciu 1966-2002

\begin{abstract}
The complete on-shell action of topological Einstein-Maxwell gravity in fourdimensions is presented. It is shown explicitly how this theory for $S U(2)$ holonomy manifolds arises from four-dimensional Euclidean $N=2$ supergravity. The twisted local BRST symmetries and twisted local Lorentz symmetries are given and the action and stress tensor are shown to be BRST-exact. A set of BRST-invariant topological operators is given. The local vector and antisymmetric tensor twisted supersymmetries and their algebra are also found.
\end{abstract}




\section{Introduction}

The study of topological gauge theories is now a large and important field in mathematical physics, yielding important insights and results in diverse dimensions. One aspect of this which has been of interest in recent years is the investigation of topological gauge theories on manifolds of special holonomy. For example, in four dimensions there is the Vafa-Witten theory, arising from $N=4, d=4$ super Yang-Mills on manifolds with $S U(2)$ holonomy [1]. In higher dimensions one also finds topological gauge theories when one considers Yang-Mills theories on manifolds of special holonomy - in eight dimensions there is a topological gauge theory defined on manifolds with $\operatorname{Spin}(7)$ holonomy and there are analogous theories on seven-dimensional $G_{2}$ holonomy manifolds and Calabi-Yau three- and four-folds [2]. These theories have a rather intriguing structure. What is more, they arise naturally in the study of wrapped Euclidean branes in string theory, providing a rich source of new insights and approaches.

It is natural to enquire if a similar set of topological gravity theories exists. This is a little harder to answer, as supergravity theories are rather more complicated. The first topological gravity, in four dimensions, was found by Witten [3]. This is based on the Lagrangian which is the square of the Weyl tensor, and the theory is defined on any Riemannian four-manifold. Topological gravities on special holonomy manifolds have yet to be fully described however. A natural theory to elucidate first is the four-dimensional topological Einstein-Maxwell gravity. This should arise from $N=2$ supergravity on manifolds with $S U(2)$ holonomy, using the covariantly constant spinors to twist the fermions. An earlier pioneering analysis of this theory was given in [4]. A number of other papers also explored this subject [5]. Recently [6], the geometric formulation of this theory was clarified, and the action given up to quadratic order in the fermions.

In this paper we present the complete on-shell formulation of this fourdimensional topological gravity, with the full local BRST symmetries. A new $S U(2)$ global R-symmetry is given and we explain how the local Lorentz group is 
twisted to have a non-standard action on the fields. We also give the twisted current multiplet and its local BRST cohomology and show explicitly that the stress tensor is exact under the local BRST symmetry. We further propose a new set of observables for $S U(2)$ holonomy manifolds. All of these results are new.

As well as these new results, our work clarifies a number of issues which have appeared opaque in previous works. The discussion of twisting in [4] involved an identification of the local Lorentz and global R-symmetry group which is clearly problematic. Our explicit use of Killing vectors on $S U(2)$ holonomy four-folds makes the correct procedure quite clear, and further leads to a theory with an explicit twisted local Lorentz symmetry, which also makes it evident that the theory being considered has the required diffeomorphism invariance. In addition, since our BRST symmetry is also local, our expressions are explicitly supercovariant, which is not obvious in some earlier works such as [4]. Also, the twisted BRSTs in this reference do not square to zero on-shell as claimed, since it is not consistent to simply drop higher order terms from the transformations - one must write the full transformations first, calculate their square, and only then consider terms order order in fermions, as we do in this paper.

The paper is organised as follows. In section 2 we consider Euclidean $N=2$, $d=4$ supergravity theory on an $S U(2)$ holonomy manifold. In section 3 we study the resulting topological gravity theory, giving the twisted local BRST symmetries and twisted local Lorentz symmetries. New residual global R-symmetries of the twisted theory are also given. We show that both the twisted action and the vierbein stress tensor are BRST-exact and we give a set of BRST invariant topological operators derived from descent equations. The complete twisted current multiplet and its cohomology are also found. In section 4 we give the other twisted supersymmetries of the theory, and we finish in section 5 with a brief discussion of the context of these results and further applications. 


\section{Twisted $N=2$ four-dimensional supergravity and manifolds with $S U(2)$ holonomy}

We begin with Euclidean $N=2, d=4$ supergravity [7]. This has as bosonic fields the vierbein $e_{\mu}^{a}$, and the graviphoton $A_{\mu}$. The fermionic fields are two symplectic Majorana gravitini which may be collected into one Dirac gravitino $\chi_{\mu}$. World indices $\mu, \nu, \ldots$ and local Lorentz indices $a, b, \ldots$ run from one to four. The Euclidean theory is invariant under the bosonic symmetry $\operatorname{Spin}(4) \times S U(2) \times \operatorname{Spin}(1,1)$ where $\operatorname{Spin}(4) \cong S U(2)_{+} \times S U(2)_{-}$is the local Lorentz group and $S U(2) \times \operatorname{Spin}(1,1)$ is the global R-symmetry group. Under the $\operatorname{Spin}(1,1)$ R-symmetry subgroup, the bosonic fields $e_{\mu}^{a}$ and $A_{\mu}$ transform with weight zero and the fermionic fields $\chi_{\mu}\left(\chi_{\mu}^{\dagger}\right)$ with weight $1 / 2(-1 / 2)$.

The supersymmetric action is given by [9]

$$
\begin{aligned}
S^{N=2}=\int d^{4} x e\left(\frac{1}{2 \kappa^{2}}\right. & R(e, \hat{\omega})+\epsilon^{\mu \nu \rho \sigma} \chi_{\mu}^{\dagger} \gamma_{\nu} \hat{D}_{\rho} \chi_{\sigma}-\frac{1}{4} F_{\mu \nu}^{2} \\
& \left.+\frac{i \kappa}{2 \sqrt{2}} \chi_{\mu}^{\dagger}\left[\left(F^{\mu \nu}+\hat{F}^{\mu \nu}\right)+\gamma_{5}\left(* F^{\mu \nu}+* \hat{F}^{\mu \nu}\right)\right] \chi_{\nu}\right) .
\end{aligned}
$$

Our spinor conventions are those of [9]. $\kappa$ is the gravitational constant and we define $* F_{\mu \nu}:=\frac{1}{2} \epsilon_{\mu \nu \rho \sigma} F^{\rho \sigma}$, with $\epsilon_{\mu \nu \rho \sigma}=e_{\mu}^{a} e_{\nu}^{b} e_{\rho}^{c} e_{\sigma}^{d} \epsilon_{a b c d}$, and $\epsilon_{a b c d}$ numerical. The twoform graviphoton field strength $F=d A . D:=D(\omega)$ is the gravitational covariant derivative with respect to the standard spin connection one-form $\omega^{a b}$, given by the solution of the equation $D e^{a} \equiv d e^{a}+\omega^{a b} \wedge e^{b}=0$. The action of this covariant derivative on a spinor $\lambda$ is given by $D \lambda \equiv d \lambda+\frac{1}{4} \omega^{a b} \gamma^{a b} \lambda$, where $\gamma_{a b}:=\gamma_{[a} \gamma_{b]}$. The action of the supercovariant derivative $\hat{D}:=D(\hat{\omega})$ on Lorentz tensors takes the

$\star$ This is contrasted with the Lorentzian theory in $3+1$ dimensions which is invariant under $\operatorname{Spin}(3,1) \times U(2)$. Both Euclidean and Lorentzian theories arise from the reduction of the Lorentzian $N=2$ supergravity in $5+1$ dimensions. This theory [8] is invariant under $\operatorname{Spin}(5,1) \times S U(2)$ and has 8 supersymmetries. The reduced Euclidean and Lorentzian theories both preserve all 8 supersymmetries in four dimensions though the Euclideanised theory obtained by Wick rotating the time component in the Lorentzian theory preserves no supersymmetries. This clarifies [4] what is meant by the initial "Euclidean" supergravity. 
same form as that of $D$, but in terms of the supercovariant spin connection one-form $\hat{\omega}^{a b}$ defined as the solution of the equation $\hat{D} e^{a} \equiv d e^{a}+\hat{\omega}^{a b} \wedge e^{b}=-\frac{\kappa^{2}}{2} \chi^{\dagger} \wedge \gamma_{5} \gamma^{a} \chi$. One can similarly write a supercovariant graviphoton field strength $\hat{F}$ which is defined below, together with the explicit expression for $\hat{\omega}^{a b}$ :

$$
\begin{aligned}
& \hat{F}_{\mu \nu}=F_{\mu \nu}-i \sqrt{2} \kappa \chi_{[\mu}^{\dagger} \gamma_{5} \chi_{\nu]}, \\
& \hat{\omega}_{\mu}^{a b}=\omega_{\mu}^{a b}-\frac{1}{4} \kappa^{2}\left(\chi_{a}^{\dagger} \gamma_{5} \gamma_{\mu} \chi_{b}+\chi_{a}^{\dagger} \gamma_{5} \gamma_{b} \chi_{\mu}-\chi_{\mu}^{\dagger} \gamma_{5} \gamma_{b} \chi_{a}-(a \leftrightarrow b)\right) .
\end{aligned}
$$

The action (2.1) is invariant under the standard general coordinate, local Lorentz and Maxwell transformations, as well as the local supersymmetry

$$
\begin{aligned}
\delta_{\epsilon} e_{\mu}^{a} & =-\kappa\left(\epsilon^{\dagger} \gamma_{5} \gamma^{a} \chi_{\mu}-\chi_{\mu}^{\dagger} \gamma_{5} \gamma^{a} \epsilon\right) \\
\delta_{\epsilon} A_{\mu} & =i \sqrt{2}\left(\epsilon^{\dagger} \gamma_{5} \chi_{\mu}-\chi_{\mu}^{\dagger} \gamma_{5} \epsilon\right) \\
\delta_{\epsilon} \chi_{\mu} & =\frac{2}{\kappa} \hat{D}_{\mu} \epsilon-\frac{i}{\sqrt{2}}\left(\hat{F}_{\mu \lambda} \gamma^{\lambda}+* \hat{F}_{\mu \lambda} \gamma^{\lambda} \gamma_{5}\right) \epsilon
\end{aligned}
$$

where $\epsilon$ is a Dirac spinor. We define chiral spinors by $\chi_{L}:=\frac{1}{2}\left(1+\gamma_{5}\right) \chi, \chi_{R}:=$ $\frac{1}{2}\left(1-\gamma_{5}\right) \chi$, with $\left(\gamma_{5}\right)^{2}=1$.

On four-manifolds with $S U(2)$ holonomy, there exist two covariantly constant chiral spinors which can be collected into a complex spinor $\theta$ which we will take to be right-handed. ${ }^{\dagger}$ We will normalise this so that $\theta^{\dagger} \theta=\frac{1}{2}$. On such manifolds, one may expand the gravitino one-form $\chi$ in terms of the twisted anticommuting one-form fields $\eta, \psi^{a}, \chi^{a b}$ as

$$
\begin{aligned}
& \chi_{R}=\eta \theta+\frac{1}{2} \chi^{a b} \gamma_{a b} \theta, \\
& \chi_{L}=\psi^{a} \gamma_{a} \theta
\end{aligned}
$$

The field $\chi^{a b}$ is self-dual (satisfying $\chi^{a b}=\frac{1}{2} \epsilon^{a b c d} \chi^{c d}$ ) due to the chirality of $\theta$. We take the fields $\eta, \chi^{a b}$ to be Hermitian, and $\psi^{a}$ to be anti-Hermitian. One may

$\dagger$ This convention implies that the holonomy of the spin connection is contained in the $S U(2)_{-}$ Lorentz subgroup, that is generated by the anti-self-dual Lorentz generators. 
similarly expand the local supersymmetry spinor parameter $\epsilon$ as

$$
\begin{aligned}
\epsilon_{R} & =\epsilon \theta+\frac{1}{2} \epsilon^{a b} \gamma_{a b} \theta, \\
\epsilon_{L} & =\epsilon^{a} \gamma_{a} \theta
\end{aligned}
$$

where the $\epsilon, \epsilon^{a}, \epsilon^{a b}$ on the right-hand sides of the equations above are anticommuting fields, with $\epsilon^{a b}$ self-dual, and $\epsilon, \epsilon^{a b}$ Hermitian, and $\epsilon^{a}$ anti-Hermitian. These fields will parametrise twisted supersymmetries in the twisted theory. We will only consider the twisted scalar, or local topological BRST, supersymmetry in the following section, turning to the other twisted supersymmetries in section 4 . Whilst the general coordinate and Maxwell symmetries do not change under the twisting, the local Lorentz symmetries of the action above, generated as usual by $\frac{1}{2} \gamma^{a b}$ on spinors, are twisted on $S U(2)$ holonomy manifolds, resulting in a non-standard action on the twisted fermion fields as we will see later.

With the above expansions of the gravitino and supersymmetry parameter, one can now derive the twisted theory on a manifold with $S U(2)$ holonomy. The resulting action is

$$
\begin{array}{rl}
S=\int d^{4} x & e\left(-\frac{1}{\kappa^{2}} \epsilon^{\mu \nu \rho \sigma} \omega_{\mu}^{+a b} e_{\nu}^{b} \omega_{\rho}^{+a c} e_{\sigma}^{c}-\frac{1}{2} \epsilon^{\mu \nu \rho \sigma}\left(D_{\mu}^{[1 / 2]} \psi_{\nu}^{a}\right)\left(\eta_{\rho} e_{\sigma}^{a}-2 \chi_{\rho}^{a b} e_{\sigma}^{b}\right)\right. \\
& -\frac{1}{2} F_{\mu \nu}^{-} F^{-\mu \nu}-\frac{1}{2} \epsilon^{\mu \nu \rho \sigma} \psi_{\mu}^{a}\left(\left(D_{\nu}^{[1 / 2]} \eta_{\rho}\right) e_{\sigma}^{a}-2\left(D_{\nu}^{[1 / 2]} \chi_{\rho}^{a b}\right) e_{\sigma}^{b}\right) \\
& \left.-\frac{i \kappa}{2 \sqrt{2}}\left(F^{-\mu \nu}+\hat{F}^{-\mu \nu}\right)\left(\eta_{\mu} \eta_{\nu}+\chi_{\mu}^{a b} \chi_{\nu}^{a b}\right)+\frac{i \kappa}{2 \sqrt{2}}\left(F^{+\mu \nu}+\hat{F}^{+\mu \nu}\right)\left(\psi_{\mu}^{a} \psi_{\nu}^{a}\right)\right)
\end{array}
$$

where $\omega_{\mu}^{ \pm a b}:=\frac{1}{2}\left(\omega_{\mu}^{a b} \pm \frac{1}{2} \epsilon^{a b c d} \omega_{\mu}^{c d}\right), F_{\mu \nu}^{ \pm}:=\frac{1}{2}\left(F_{\mu \nu} \pm * F_{\mu \nu}\right)$ and $D^{[1 / 2]}:=D\left(\frac{\omega+\hat{\omega}}{2}\right)$. The pure gravitational and Maxwell kinetic terms are unaffected by the twist but have been rewritten using the identities

$$
\begin{aligned}
\frac{e}{2} R(e, \omega) & \equiv-e \epsilon^{\mu \nu \rho \sigma} \omega_{\mu}^{+a b} e_{\nu}^{b} \omega_{\rho}^{+a c} e_{\sigma}^{c}+\partial_{\mu}\left(e \epsilon^{\mu \nu \rho \sigma} \omega_{\nu}^{+a b} e_{\rho}^{a} e_{\sigma}^{b}\right) \\
\frac{e}{4} F_{\mu \nu} F^{\mu \nu} & \equiv \frac{e}{2} F_{\mu \nu}^{-} F^{-\mu \nu}+\partial_{\mu}\left(\frac{e}{4} \epsilon^{\mu \nu \rho \sigma} A_{\nu} F_{\rho \sigma}\right)
\end{aligned}
$$

where $R^{a b}:=R^{a b}(\omega)=d \omega^{a b}+\omega^{a c} \wedge \omega^{c b}$ is the two-form Riemann tensor. The total derivative terms in (2.7) have been discarded in the integral (2.6). 
Twisting the supersymmetry leads to the local BRST symmetry

$$
\begin{aligned}
\delta_{\epsilon} e_{\mu}^{a} & =\kappa \epsilon \psi_{\mu}^{a}, \\
\delta_{\epsilon} A_{\mu} & =-i \sqrt{2} \epsilon \eta_{\mu}, \\
\delta_{\epsilon} \eta_{\mu} & =\frac{2}{\kappa} \partial_{\mu} \epsilon, \\
\delta_{\epsilon} \psi_{\mu}^{a} & =-i \sqrt{2} \epsilon \hat{F}_{\mu \nu}^{+} e_{a}^{\nu}, \\
\delta_{\epsilon} \chi_{\mu}^{a b} & =\frac{1}{\kappa} \epsilon \hat{\omega}_{\mu}^{+a b} .
\end{aligned}
$$

We will study this twisted theory in the following section, where we will show that it defines a topological gravity theory of cohomological type.

\section{Topological four-dimensional gravity}

In the previous section we showed how Euclidean $N=2, d=4$ supergravity on an $S U(2)$ holonomy manifold becomes twisted, giving a theory with anticommuting one-form fields $\eta, \psi^{a}$ and $\chi^{a b}$ (a self-dual Lorentz two-form), together with the commuting one-form fields $e^{a}$ and $A$. In a similar manner, the supersymmetry generator of the untwisted theory, which is a Dirac spinor, reduces to a set of zero-form fermionic generators $\left(Q, Q^{a}, Q^{a b}\right)$ (where $Q^{a b}$ is a self-dual Lorentz two-form). We will consider here the scalar BRST-like supersymmetry $Q$. The bosonic symmetries consist of the standard general coordinate and Maxwell gauge transformations which remain unchanged under the twist together with the local Lorentz transformations whose action on the anti-commuting fields becomes modified in the twisted theory, and further one has a rigid $S U(2)$ R-symmetry, and an Abelian symmetry of the equations of motion, as we will see.

It will prove convenient now to set $\kappa=2$ and to redefine the fields by $e^{a} \rightarrow$ $i \sqrt{2} e^{a}, A \rightarrow-i \sqrt{2} A, \psi^{a} \rightarrow \frac{i}{\sqrt{2}} \psi^{a}$. It is also convenient to introduce the second rank Lorentz tensor-valued one-form quantity $T^{a b}:=\delta^{a b} \eta-2 \chi^{a b^{\star}}$. Written in

\footnotetext{
* Since each component of $T^{a b}$ is in an irreducible representation of $S O(4)$ then one can reobtain the fields $\eta$ and $\chi^{a b}$ by Young projection on $T^{a b}$.
} 
terms of differential forms, the action (2.6) and BRST symmetry (2.8) then become (we drop an overall factor coming from the scaling of the integration measure under the above field redefinitions)

$$
\begin{aligned}
S=\int & \left(\frac{1}{2}\left(\omega^{+a b} \wedge e^{b}\right) \wedge\left(\omega^{+a c} \wedge e^{c}\right)-2 F^{-} \wedge F^{-}\right. \\
& -\frac{1}{2} T^{a b} \wedge e^{b} \wedge D^{[1 / 2]} \psi^{a}+\frac{1}{2} \psi^{a} \wedge e^{b} \wedge D^{[1 / 2]} T^{a b} \\
+ & \left.\frac{1}{4}(F+\hat{F})^{-} \wedge T^{a b} \wedge T^{a b}+\frac{1}{2}(F+\hat{F})^{+} \wedge \psi^{a} \wedge \psi^{a}\right)
\end{aligned}
$$

and

$$
\begin{aligned}
\delta_{\epsilon} e^{a} & =\epsilon \psi^{a}, \\
\delta_{\epsilon} A & =\epsilon \eta, \\
\delta_{\epsilon} \eta & =d \epsilon, \\
\delta_{\epsilon} \psi^{a} & =-2 \epsilon \hat{F}^{-a b} e^{b}, \\
\delta_{\epsilon} \chi^{a b} & =\frac{1}{2} \epsilon \hat{\omega}^{+a b} .
\end{aligned}
$$

World and Lorentz indices are related using vierbein $e_{\mu}^{a}$, with $F_{a b}=F_{\mu \nu} e_{a}^{\mu} e_{b}^{\nu}$. The differential form notation is introduced here for simplicity.

The local Lorentz symmetries are given by twisting the original local Lorentz transformations, giving

$$
\begin{aligned}
\delta_{L} e^{a} & =\Lambda^{a b} e^{b}, \\
\delta_{L} A & =0 \\
\delta_{L} \eta & =-\frac{1}{2} \Lambda^{+a b} \chi^{a b}, \\
\delta_{L} \psi^{a} & =\Lambda^{-a b} \psi^{b}, \\
\delta_{L} \chi^{a b} & =\frac{1}{2} \Lambda^{+a b} \eta+\Lambda^{+c[a} \chi^{b] c},
\end{aligned}
$$

for antisymmetric local Lorentz parameter $\Lambda^{a b}(x)$. The composite field $T^{a b}$ transforms nicely under these twisted Lorentz transformations with $\delta_{L} T^{a b}=\Lambda^{+b c} T^{a c}$. 
Notice how the self-dual part of the local Lorentz parameter $\Lambda^{a b}$ acts only on $\eta$ and $\chi^{a b}$, whilst $\psi^{a}$ transforms only under the anti-self-dual part (as expected, since the action is localised on self-dual connections and $\psi^{a}$ corresponds to vierbein variations preserving this condition [6]).

The associated covariant derivatives $D$ can be written schematically as $D[-]=$ $d[-]+\delta_{L}(\omega) \wedge[-]$, so that

$$
\begin{aligned}
D \eta & =d \eta-\frac{1}{2} \omega^{+a b} \wedge \chi^{a b}, \\
D \psi^{a} & =d \psi^{a}+\omega^{-a b} \wedge \psi^{b}, \\
D \chi^{a b} & =d \chi^{a b}+\frac{1}{2} \omega^{+a b} \wedge \eta+\omega^{+c[a} \wedge \chi^{b] c} .
\end{aligned}
$$

The action (3.1) has a further rigid $S U(2)$ R-symmetry, given by

$$
\begin{aligned}
\delta_{\lambda} e^{a} & =\lambda^{+a b} e^{b}, \\
\delta_{\lambda} A & =0, \\
\delta_{\lambda} \eta & =0, \\
\delta_{\lambda} \psi^{a} & =\lambda^{+a b} \psi^{b}, \\
\delta_{\lambda} \chi^{a b} & =\lambda^{+c[a} \chi^{b] c},
\end{aligned}
$$

where $\lambda^{+a b}$ is the constant self-dual parameter of the symmetry. This symmetry is independent from the local Lorentz invariance under (3.3) with global parameter $\Lambda^{a b}(x)=\Lambda^{a b}$. Note however that the transformations corresponding to (3.5) with a constant anti-self-dual parameter $\lambda^{-a b}$ are a special case of the local Lorentz transformations, with $\Lambda^{a b}=\lambda^{-a b}$.

The supercovariant and local Lorentz invariant graviphoton field strength $\hat{F}$ in (3.1), (3.2) is given by

$$
\hat{F}=F-\frac{1}{8} T^{a b} \wedge T^{a b}+\frac{1}{4} \psi^{a} \wedge \psi^{a} .
$$

The supercovariant spin connection $\hat{\omega}(e, T, \psi)$ is the solution of the equation $\hat{D} e^{a} \equiv$ 
$(\hat{\omega}-\omega)^{a b} \wedge e^{b}=T^{b a} \wedge \psi^{b}$. The explicit solution is given by

$$
\hat{\omega}_{\mu}^{a b}=\omega_{\mu}^{a b}-2 e^{\nu[a} T_{[\mu}^{|c| b]} \psi_{\nu]}^{c}+e^{\rho a} e^{\sigma b} e_{\mu}^{c} T_{[\rho}^{d c} \psi_{\sigma]}^{d} .
$$

The supercovariant objects just defined follow from twisting the corresponding objects in the previous section. We note in passing the result

$$
\left(D^{[1 / 2]} \psi^{a}\right) \wedge T^{a b} \wedge e^{b}=\psi^{a} \wedge\left(D^{[1 / 2]} T^{a b}\right) e^{b}-\frac{1}{16} T^{a b} \wedge T^{a b} \wedge \psi^{c} \wedge \psi^{c}
$$

up to a total derivative, implying that terms quartic in the fermions are generated in the twisted theory if one writes the action with only left- or right- acting covariant derivatives in the fermion kinetic terms.

The explicit BRST-supercovariant form of the twisted action and symmetries above clarifies the results in [4] and the non-standard geometrical symmetries (3.3), (3.5) are new.

It should be noted that once having used the $S U(2)$ structure to twist, the resulting action (3.1) is invariant under all the transformations above on any Riemannian four-manifold. Writing this twisted theory on a general four-manifold however necessitates a non-standard action of the local Lorentz group on fields (3.3), (3.4) in order to maintain covariance.

We will now show that the model detailed above satisfies the axioms of a topological field theory of cohomological type. That is, the action of the twisted scalar BRST operator $Q$ forms an on-shell, (Lorentz + gauge) equivariant cohomology in which the action functional is closed and the associated stress tensor is exact. It will also be shown that the action functional itself is BRST-exact. 


\subsection{BRST INVARIANCE OF THE ACTION}

This follows from the supersymmetry of the untwisted theory, nevertheless an explicit calculation is a useful check of the result. The following procedure simplifies the calculation. Writing the set of one-form fields in the twisted theory as $\{\Phi\}$ then it is evident that under an arbitrary infinitesimal field transformation $\delta \Phi$, the action $S$ varies as

$$
\delta S=\int \sum_{\Phi}(\delta \Phi) \wedge J_{\Phi}
$$

where all total derivative terms are dropped. $\left\{J_{\Phi}\right\}$ form a set of three-forms associated with $\{\Phi\}$. Each of these three-forms are Hodge-dual to the equations of motion for the respective fields in the sense that $[\Phi]:=\frac{\delta S}{\delta \Phi}=* J_{\Phi}=0$ is the field equation for $\Phi$.

To lowest order in the fermionic fields, it is found that under the local BRST transformations (3.2), the $\left[e^{a}\right]$ terms in the variation of the twisted action (3.1) cancel all the $\left[T^{a b}\right]$ terms and also various $\left[\psi^{a}\right]$ terms. The $[A]$ terms cancel the remaining $\left[\psi^{a}\right]$ terms. The action (3.1) is also found to be invariant under (3.2) to all orders with various additional cross-cancellations occuring.

The fact that this calculation holds for local BRST transformations can be seen from the fact that the three-form field equation tensors

$$
\begin{aligned}
J_{e^{a}} & =-e^{b} \wedge R^{a b}\left(\hat{\omega}^{+}\right)-\psi^{b} \wedge \hat{D} T^{b a}-4 \hat{F}^{-a b} e^{b} \wedge \hat{F}^{+}, \\
J_{A} & =-d\left(4 \hat{F}^{-}-\psi^{a} \wedge \psi^{a}\right), \\
J_{T^{a b}} & =-e^{b} \wedge \hat{D} \psi^{a}+T^{a b} \wedge \hat{F}^{-}, \\
J_{\psi^{a}} & =e^{b} \wedge \hat{D} T^{a b}+2 \psi^{a} \wedge \hat{F}^{+},
\end{aligned}
$$

are explicitly BRST-supercovariant. 
The equations of motion (3.6) are invariant under the transformations

$$
\begin{aligned}
\delta_{\alpha} e^{a} & =0, \\
\delta_{\alpha} \hat{F}^{-} & =-2 \alpha \hat{F}^{-}, \\
\delta_{\alpha} \hat{F}^{+} & =2 \alpha \hat{F}^{+}, \\
\delta_{\alpha} \eta & =\alpha \eta, \\
\delta_{\alpha} \psi^{a} & =-\alpha \psi^{a}, \\
\delta_{\alpha} \chi^{a b} & =\alpha \chi^{a b},
\end{aligned}
$$

for real parameter $\alpha$. These BRST-supercovariant transformations define the (nonlocal in $A$ ) transformations of $F^{ \pm}$.

Some identities used above and in various calculations throughout this paper are

$$
\begin{aligned}
& A_{a c}^{-} B_{b c}^{+}=A_{b c}^{-} B_{a c}^{+}, \\
& A_{a c}^{ \pm} B_{b c}^{ \pm}+A_{b c}^{ \pm} B_{a c}^{ \pm}=\frac{1}{2} \delta_{a b} A_{c d}^{ \pm} B_{c d}^{ \pm}, \\
& A_{a e}^{ \pm} B_{b e}^{ \pm}-A_{b e}^{ \pm} B_{a e}^{ \pm} \mp \epsilon_{a b c d} A_{c e}^{ \pm} B_{d e}^{ \pm}=0
\end{aligned}
$$

for any antisymmetric tensors $A_{a b}, B_{a b}$.

Note also that the tensor $\epsilon_{\mu \nu \rho \sigma}$ depends on the vierbein. This results in additional variations of the vierbein when one varies the (anti-)self-dual parts $A_{\mu \nu}^{ \pm}$of a two-form $A_{\mu \nu}$. If the variation of $A$ is $\delta A$ then

$$
\delta\left(A^{ \pm}\right)=(\delta A)^{ \pm}-\left(\delta e^{a} \wedge e^{b}\right)^{ \pm} A_{a b}^{\mp},
$$

where $A_{a b}=A_{\mu \nu} e_{a}^{\mu} e_{b}^{\nu}$. 


\subsection{Nilpotence of the BRST Symmetry}

The local BRST transformations (3.2) are equivariantly nilpotent on-shell, satisfying $\left[\delta_{\epsilon}, \delta_{\epsilon^{\prime}}\right]=0$ up to fermionic equations of motion, local Lorentz transformations and $U(1)$ gauge transformations. The local Lorentz transformations

have parameter $\Lambda^{a b}=4 \epsilon \epsilon^{\prime} \hat{F}^{-a b}$, whilst the gauge transformations have parameter $-\epsilon \epsilon^{\prime}$. The proof of BRST nilpotence requires showing that $\delta_{\epsilon} \hat{\omega}^{+a b}=0$ and $\left(\delta_{\epsilon} \hat{F}^{-a b}\right) e^{b}=0$ on-shell. It is straightforward but rather tedious to show that these equations are true. This involves taking various contractions of the $\eta, \psi^{a}$ and $\chi^{a b}$ equations of motion with the inverse vierbein. For example, taking contractions of the vierbien with the $\psi^{a}$ equation of motion, one deduces that

$$
\left.\delta_{\epsilon}\left(F_{\mu \nu}-\eta_{\mu} \eta_{\nu}-\chi_{\mu}^{a b} \chi_{\nu}^{a b}\right)\right|_{[\mu \nu]-}=\left.2 \epsilon\left(\hat{D}_{[\mu} \eta_{\nu]}+e_{(\mu}^{a} \psi_{\lambda)}^{a} \hat{F}_{\nu}^{+\lambda}\right)\right|_{[\mu \nu]-}=0
$$

on-shell. This result is used to prove that the BRST symmetry is nilpotent when acting on $\psi^{a}$. More complicated manipulations of contractions of the vierbein with the $\eta$ and $\chi^{a b}$ equations of motion yield the result that

$$
\left.\left(e^{\nu a} \hat{D}_{[\nu} \psi_{\mu]}^{b}+\hat{F}_{\mu \nu}^{-} e^{\nu a} e^{\rho b} \eta_{\rho}-2 \hat{F}_{\mu \nu}^{-} e^{\nu c} e^{\rho b} \chi_{\rho}^{c a}\right)\right|_{[a b]}=0
$$

on-shell. This result is used to prove that the BRST symmetry is nilpotent when acting on $\chi^{a b}$. Note that this calculation only works by using the full supercovariant BRST rules (3.2). The lowest order on-shell global BRST transformations given in (4.3) of [4] do not square to zero (even to lowest order on-shell and up to local transformations). 


\subsection{EXACTNESS OF THE ACTION}

Not only is the action (3.1) BRST-closed, it is also BRST-exact, up to the fermion equations of motion. This is in the sense that if one writes the action $S=\int d^{4} x L$ then one has $\int d^{4} x \epsilon L=\delta_{\epsilon} \Psi$, where the gauge fermion $\Psi$ is given by

$$
\begin{aligned}
\Psi=\int & \left(\chi^{a b} \wedge e^{b} \wedge\left(\hat{\omega}^{a c} \wedge e^{c}-2 \eta \wedge \psi^{a}\right)\right. \\
& \left.+\frac{1}{2} e^{a} \wedge \psi^{a} \wedge\left(\hat{F}^{-}+\frac{1}{4} T^{b c} \wedge T^{b c}-\frac{1}{4} \psi^{b} \wedge \psi^{b}\right)\right) .
\end{aligned}
$$

This is fairly straightforward to prove if one notes that since one is allowed to use the fermion equations of motion, one can use these to generate the terms in the action which are quadratic in the fermions. This will also generate other terms quartic in the fermions. Combining these with the results of performing the BRST variation of the gauge fermion above, one proves that precisely the action (3.1) is generated. Note also that one assumes that the BRST parameter $\epsilon$ is constant for this calculation. Any terms involving $d \epsilon$ can be absorbed as total derivatives into the Lagrangian.

It should be noted that the gauge fermion above is invariant under the $\Lambda^{-a b}$ part of the local Lorentz transformation $\delta_{L}\left(\Lambda^{a b}\right)$, but not under the $\Lambda^{+a b}$ part. This is consistent with localisation in the path integral. That is, one can introduce a coupling constant in front of the Lagrangian, and use exactness of the action to show that correlation functions are independent of the value of this coupling constant. This can be used to prove that the path integral localises on field configurations with $\omega^{+a b} \wedge e^{b}=0$ and $F^{-}=0$. The former is only invariant under the $\Lambda^{-a b}$ local Lorentz transformations.

In the gravitational sector, localisation on vierbeins satisfying the anti-selfdual spin connection constraint $\omega_{\mu}^{+a b}=0$ implies localisation on metrics satisfying the constraint $R^{a b}+\frac{1}{2} \epsilon^{a b c d} R^{c d}=0$, due to the identity $R^{a b}\left(\omega^{+}\right)=R^{+a b}(\omega)$. Gravitational instanton metric solutions of this equation have been well studied. 
Note that under the standard Kaluza-Klein reduction of the four-dimensional metric $g_{\mu \nu} \rightarrow\left(e^{\phi / \sqrt{3}} g_{i j}+e^{-2 \phi / \sqrt{3}} A_{i} A_{j}, e^{-2 \phi / \sqrt{3}} A_{i}, e^{-2 \phi / \sqrt{3}}\right)$ to three dimensions, this anti-self-duality condition reduces to two independent, covariant constraints. The first just demands that one considers flat spin connections in three dimensions whilst the second imposes the non-linear Bogomolnyi type equation

$$
F_{i j}+e^{\phi} \epsilon_{i j k} \partial^{k} \phi=0
$$

where $F_{i j}=2 \partial_{[i} A_{j]}$ and $\epsilon_{i j k}$ denotes the orientation of the three-manifold. This is analogous to the reduction of Donaldson-Witten theory where one obtains Floer theory in three dimensions whose path integral localises on solutions of the standard Bogomolnyi equation. The topological gravity in three dimensions which localises on solutions of (3.9) would analogously be obtained by simple Kaluza-Klein reduction of the theory presented here.

\subsection{EXACTNESS OF THE STRESS TENSOR}

The action (3.1) is a functional of the vierbien $e_{\mu}^{a}$, explicitly as well as through its dependence on the metric $g_{\mu \nu}=e_{\mu}^{a} e_{\nu}^{a}$. Variation of the action with respect to the (inverse) vierbein generates the "vierbein stress tensor" *

$$
V_{\mu}^{a}=\frac{1}{e} \frac{\delta S}{\delta e_{a}^{\mu}} \equiv \frac{1}{e}\left[e_{\mu}^{a}\right]
$$

Thus the vierbein stress tensor is given (up to a factor of e) by the equation of motion for the vierbein. The expression for $V^{a}$ (as a one-form) then simply follows from $\left[e^{a}\right]=* J_{e^{a}}$ defined in (3.6).

$\star$ This is related to the usual stress tensor $T_{\mu \nu}=\frac{2}{\sqrt{g}} \frac{\delta S}{\delta g^{\mu \nu}}$ by $T_{\mu \nu}=e_{\nu}^{a} V_{\mu}^{a}$. Conservation and symmetry of the stress tensor follow from the general coordinate and local Lorentz invariance of the action (see [10]). 
Since the BRST transformations are a symmetry of the theory, they must take equations of motion into other equations of motion. In particular, a calculation shows that the BRST variation of the field equation three-forms (3.6) give

$$
\begin{aligned}
\delta_{\epsilon} J_{\psi^{a}} & =\epsilon J_{e^{a}}, \\
\delta_{\epsilon} J_{\eta} & =\epsilon J_{A}, \\
\delta_{\epsilon} J_{\chi^{a b}} & =0 .
\end{aligned}
$$

These relations follow even for local BRST transformations since the field equations (3.6) are supercovariant. The implication of (3.10) is that the stress tensor is BRST-exact, on-shell, such that

$$
\epsilon T_{\mu \nu}=\delta_{\epsilon} G_{\mu \nu}
$$

for some function $G_{\mu \nu}$. This statement follows since the vierbein stress tensor $V_{\mu}^{a}=T_{\mu \nu} e^{\nu a}=\frac{1}{e}\left(* J_{e^{a}}\right)_{\mu}^{a}$. Therefore, using (3.10) and the fact the $* J_{\psi^{a}}=0$ is a fermionic equation of motion, one has the result (3.11). Thus the local BRST transformations provide the twisted theory with a cohomological structure.

In the off-shell, gauge-fixed formulation of twisted gauge theories then exactness of the stress tensor follows from exactness of the action. This is why the calculation above is not done in [4]. For twisted gravitational theories though, gauge-fixing implies fixing diffeomorphism symmetry and then it is not so clear whether the statement above is still valid.

\subsection{Descent equations and invariants}

To construct a basis of BRST-invariant topological operators, consider a set of operators of the form $\mathcal{O}_{i}=\int_{\gamma_{i}} W_{(i)}$ (where $\gamma_{i}$ is an $i$-cycle in homology and $W_{(i)}$ is an $i$-form). These operators are constructed in the standard way, via the descent equations $Q \cdot W_{(i+1)}=d W_{(i)}^{\dagger}{ }^{\dagger}$ These equations are solved by starting

\footnotetext{
$\dagger$ We here consider rigid BRST transformations $\delta_{\epsilon}(3.2)$, which can be factorised such that $\delta_{\epsilon}=\epsilon Q$, defining the action of $Q$ on the fields.
} 
with a four-form characteristic class $W_{(4)}$ whose evaluation on the four-manifold produces a topological invariant. Recall that for twisted gauge theories in four dimensions, this is given by the second Chern class of the gauge bundle which is proportional to $F \wedge F$ and whose evaluation gives the instanton number. For twisted gravity in four dimensions there are two natural four-form characteristic classes on the tangent bundle of the four-manifold. These are the first Pontrjagin class (proportional to $R^{a b} \wedge R^{a b}$ ) and the Euler class (proportional to $\epsilon^{a b c d} R^{a b} \wedge$ $R^{c d}$ ) whose evaluation gives the Hirzebruch signature and Euler number invariants, respectively. It is convenient to take linear combinations of these two classes to define $W_{(4)}^{ \pm}:=R^{ \pm a b} \wedge R^{ \pm a b}$. The set of descendent BRST-invariant operators can then be written

$$
\begin{aligned}
& \mathcal{O}_{4}^{ \pm}=\int_{\gamma_{4}} R^{ \pm a b} \wedge R^{ \pm a b} \\
& \mathcal{O}_{3}^{ \pm}=\int_{\gamma_{3}} 2 N^{ \pm a b} \wedge R^{ \pm a b} \\
& \mathcal{O}_{2}^{+}=\int_{\gamma_{2}} N^{+a b} \wedge N^{+a b} \\
& \mathcal{O}_{2}^{-}=\int_{\gamma_{2}}\left(4 \hat{F}^{-a b} R^{-a b}+N^{-a b} \wedge N^{-a b}\right), \\
& \mathcal{O}_{1}^{-}=\int_{\gamma_{1}} 4 \hat{F}^{-a b} N^{-a b} \\
& \mathcal{O}_{0}^{-}=\int_{\gamma_{0}} 4 \hat{F}^{-a b} \hat{F}^{-a b} .
\end{aligned}
$$

The anticommuting Lorentz two-form valued one-form $N^{a b}:=Q \cdot \omega^{a b}$ and $\mathcal{O}_{1}^{+}=$ $\mathcal{O}_{0}^{+}=0$. Each operator in (3.12) is $Q$-invariant, though for $\mathcal{O}_{2}^{ \pm}, \mathcal{O}_{1}^{-}$and $\mathcal{O}_{0}^{-}$this requires the fermionic field equation $\left(Q \cdot \hat{F}^{-a b}\right) e_{\mu}^{b}=0$. These BRST supercovariant operators are related to those proposed in [4] after identifying $\hat{F}^{a b}$ and $N^{a b}$ with their appropriate ghost fields, called $\eta_{0}{ }^{a b}$ and $\chi_{0}{ }^{a b}$ respectively in [4].

There is also the Chern class of the gauge bundle from which one can construct 
topological operators. The associated BRST-invariant operators are

$$
\begin{aligned}
& \mathcal{O}_{4}^{\prime}=\int_{\gamma_{4}} F \wedge F, \\
& \mathcal{O}_{3}^{\prime}=\int_{\gamma_{3}} 2 \eta \wedge F, \\
& \mathcal{O}_{2}^{\prime}=\int_{\gamma_{2}} \eta \wedge \eta,
\end{aligned}
$$

which are $Q$-invariant off-shell.

We note that, up to boundary terms, the integrands in $\mathcal{O}_{3}^{ \pm}, \mathcal{O}_{2}^{+}$and $\mathcal{O}_{1}^{-}$are $Q$ exact (for $\mathcal{O}_{1}^{-}$this uses the fermionic field equation $\left(Q \cdot \hat{F}^{-a b}\right) e_{\mu}^{b}=0$ ). For example, the BRST variations of the Chern-Simons three-forms for $\omega^{ \pm a b}$ yield the integrands in $\mathcal{O}_{3}^{ \pm}$up to a total derivative. The integrands in $\mathcal{O}_{2}^{\prime}$ and $\mathcal{O}_{3}^{\prime}$ are also $Q$-exact. We remark that $S U(2)$ holonomy manifolds are necessarily Ricci-flat. Thus, for these manifolds, using (2.7), $\omega^{+a b} \wedge e^{b} \wedge \omega^{+a c} \wedge e^{c}=d\left(\omega^{+a b} \wedge e^{a} \wedge e^{b}\right)$, so that locally this term in the Lagrangian is a total derivative. It is therefore potentially a four-form characteristic class on four-manifolds with holonomy in $S U(2)$ that would lead to a new set of descendent topological observables.

\section{Further twisted supersymmetries}

The topological gauge theory presented here has been shown to have local BRST invariance. However, as can been seen from (2.5), twisting the supersymmetry of the original Euclidean $N=2, d=4$ supergravity would then be expected to yield further local fermionic symmetries. This is the case, and one has a vector 
supersymmetry, with anti-commuting parameter $\epsilon^{a}$, acting as

$$
\begin{aligned}
\delta_{\epsilon^{a}} e^{a} & =\epsilon^{b} T^{b a}, \\
\delta_{\epsilon^{a}} A & =-\frac{1}{2} \epsilon^{a} \psi^{a}, \\
\delta_{\epsilon^{a}} \eta & =\epsilon^{a} \hat{F}^{+a b} e^{b}, \\
\delta_{\epsilon^{a}} \chi^{a b} & =-2 \hat{F}^{+c[a} \epsilon^{b]+} e^{c}, \\
\delta_{\epsilon^{a}} \psi^{a} & =d \epsilon^{a}+\hat{\omega}^{-a b} \epsilon^{b} \equiv \hat{D} \epsilon^{a} .
\end{aligned}
$$

There is also an antisymmetric tensor supersymmetry, with anti-commuting, selfdual parameter $\epsilon^{a b}$, acting as

$$
\begin{aligned}
\delta_{\epsilon^{a b}} e^{a} & =2 \epsilon^{a b} \psi^{b}, \\
\delta_{\epsilon^{a b}} A & =\epsilon^{a b} \chi^{a b} \\
\delta_{\epsilon^{a b}} \eta & =-\frac{1}{2} \epsilon^{a b} \hat{\omega}^{+a b}, \\
\delta_{\epsilon^{a b}} \chi^{a b} & =d \epsilon^{a b}+\hat{\omega}^{+c[a} \epsilon^{b] c} \equiv \hat{D} \epsilon^{a b}-\frac{1}{2} \epsilon^{a b} \eta, \\
\delta_{\epsilon^{a b}} \psi^{a} & =4 \epsilon^{a b} \hat{F}^{-b c} e^{c} .
\end{aligned}
$$

The supersymmetry algebra of Euclidean $N=2, d=4$ supergravity closes onshell, modulo (field-dependent) diffeomorphisms, local Lorentz transformations, gauge transformations and supersymmetries. This is reflected in the brackets of the twisted supersymmetries $\left(\delta_{\epsilon}, \delta_{\epsilon^{a}}, \delta_{\epsilon^{a b}}\right)$ and Lorentz transformations $\delta_{L}$, given by 


$$
\begin{aligned}
& {\left[\delta_{\epsilon}, \delta_{\epsilon^{\prime}}\right]=\delta_{L}\left(4 \epsilon \epsilon^{\prime} \hat{F}^{-a b}\right)+\delta_{\text {gauge }}\left(-\epsilon \epsilon^{\prime}\right),} \\
& {\left[\delta_{\epsilon^{a}}, \delta_{\epsilon^{\prime a}}\right]=\delta_{L}\left(-2 \epsilon^{c} \epsilon^{\prime c} \hat{F}^{+a b}\right)+\delta_{\text {gauge }}\left(\frac{1}{2} \epsilon^{a} \epsilon^{\prime a}\right),} \\
& {\left[\delta_{\epsilon^{a b}}, \delta_{\epsilon^{\prime a b}}\right]=\delta_{L}\left(4 \epsilon^{c d} \epsilon^{\prime c d} \hat{F}^{-a b}\right)+\delta_{\text {gauge }}\left(-\epsilon^{a b} \epsilon^{\prime a b}\right),} \\
& {\left[\delta_{\epsilon}, \delta_{\epsilon^{\prime a}}\right]=\delta_{L}\left(-\epsilon \epsilon^{\prime \mu} \hat{\omega}_{\mu}^{a b}\right)+\mathcal{L}_{\left(-\epsilon \epsilon^{\prime \mu}\right)}+\delta_{\text {gauge }}\left(\epsilon \epsilon^{\prime \mu} A_{\mu}\right)} \\
& +\delta_{\left(\epsilon \epsilon^{\prime \mu} \eta_{\mu}\right)}+\delta_{\left(\epsilon \epsilon^{\prime \mu} \chi_{\mu}^{a b}\right)}+\delta_{\left(\epsilon \epsilon^{\prime \mu} \psi_{\mu}^{a}\right)} \\
& {\left[\delta_{\epsilon}, \delta_{\epsilon^{\prime a b}}\right]=0 \text {, }} \\
& {\left[\delta_{\epsilon^{a}}, \delta_{\epsilon^{\prime a b}}\right]=\delta_{L}\left(2 \epsilon^{c} \epsilon^{\prime c \mu} \hat{\omega}_{\mu}^{a b}\right)+\mathcal{L}_{\left(2 \epsilon^{a} \epsilon^{\prime a \mu}\right)}+\delta_{\text {gauge }}\left(-2 \epsilon^{a} \epsilon^{\prime a \mu} A_{\mu}\right)} \\
& +\delta_{\left(-2 \epsilon^{a} \epsilon^{\prime a \mu} \eta_{\mu}\right)}+\delta_{\left(-2 \epsilon^{c} \epsilon^{\prime c \mu} \chi_{\mu}^{a b}\right)}+\delta_{\left(-2 \epsilon^{b} \epsilon^{\prime b \mu} \psi_{\mu}^{a}\right)}, \\
& {\left[\delta_{\epsilon}, \delta_{L}\left(\Lambda^{a b}\right)\right]=\delta_{\left(\frac{1}{2} \epsilon \Lambda^{+a b}\right)},} \\
& {\left[\delta_{\epsilon^{a}}, \delta_{L}\left(\Lambda^{a b}\right)\right]=\delta_{\left(\epsilon^{b} \Lambda^{-a b}\right)},} \\
& {\left[\delta_{\epsilon^{a b}}, \delta_{L}\left(\Lambda^{a b}\right)\right]=\delta_{\left(-\frac{1}{2} \epsilon^{a b} \Lambda^{+a b}\right)}+\delta_{\left(-\epsilon^{c[a} \Lambda^{+b] c}\right)} .}
\end{aligned}
$$

The notation in the right hand side of (4.3) is that $\mathcal{L}_{k^{\mu}}$ denotes the Lie derivative of a tensor under an infinitesimal general coordinate transformation $\delta x^{\mu}=-k^{\mu}$, whilst $\delta_{\text {gauge }}(\xi)$ denotes an infinitesimal $U(1)$ gauge transformation with parameter $\xi$. World and Lorentz indices are interchanged using the vierbein and its inverse.

Typically these other twisted supersymmetries are not preserved for twisted gauge theories on a general curved background and are not given in [4] though for twisted supergravities they are a well-defined symmetry.

\section{Discussion}

We have presented here the complete on-shell action and symmetries for topological four-dimensional Einstein-Maxwell gravity, together with a set of associated topological operators. Topological gravities on higher-dimensional special holonomy manifolds are expected to take a similar form to the theory discussed here on manifolds of $S U(2)$ holonomy. This was conjectured in [6], and recently an eight-dimensional $\operatorname{Spin}(7)$ model has been analysed in [11]. We can thus expect that all the special holonomy topological gravities will be found soon. This will 
fill out the analogy with Yang-Mills theories and their associated special holonomy topological gauge theories. The special holonomy topological Yang-Mills theories arise as the effective theories on Euclidean branes wrapped on special holonomy manifolds. (More generally these topological theories should be of Born-Infeld type, although this formulation is only known for the Abelian Donaldson-Witten theory [12]). The way in which these new topological gravities might arise in string theory is an interesting issue. They may be realised as low energy effective actions for twisted string theories, for example. This may occur in the context of duality, since there are examples or conjectured cases where topological gravities or string theories arise from holographic dualities. Three-dimensional Chern-Simons theory is known to be dual to a topological string theory (see [13] and references therein). Also, a recently discovered topological conformal field theory in four dimensions is conjectured to be dual, via de Sitter holography, to a five-dimensional topological phase of type $I I B^{*}, d=9+1$ supergravity in the 't Hooft limit [14]. The latter is expected to have, as boundary theory, a twisted version of four-dimensional $N=4$ Euclidean conformal supergravity. The special holonomy topological gravities, whilst of interest in themselves, may also find a natural setting in string theory.

\section{REFERENCES}

1. C. Vafa and E. Witten, Nucl. Phys. B431 (1994) 77.

2. L. Baulieu, H. Kanno and I. Singer, Commun. Math. Phys. 194 (1998) 149; L. Baulieu, A. Losev and N. Nekrasov, Nucl. Phys. B522 (1998) 82; L. Baulieu and P. West, Phys. Lett. B436 (1998) 97-107; M. Blau and G. Thompson, Phys. Lett. B415 (1997) 242; B.S. Acharya, M. O'Loughlin and B. Spence, Nucl. Phys. B503 (1997) 657; B.S. Acharya, J.M. FigueroaO'Farrill, M. O'Loughlin and B. Spence, Nucl. Phys. B514 (1998) 583; J.M. Figueroa-O'Farrill, A. Imaanpur, J. McCarthy, Phys. Lett. B419 (1998) 167.

3. E. Witten, Phys. Lett. B206 (1988) 601. 
4. D. Anselmi and P. Fre, Nucl. Phys. B392 (1993) 401.

5. D. Anselmi, Class. Quant. Grav. 14 (1997) 2031-2047; M. Abe, Mod. Phys. Lett A12 (1997) 381-392; P.L. Paul, Topological Symmetries of Twisted N=2 Chiral Supergravity in Ashtekar Formalism, hep-th/9504144.

6. L. Baulieu and A. Tanzini, JHEP 03 (2002) 015.

7. S. Ferrara and P. van Nieuwenhuizen, Phys. Rev. Lett. 3725 (1976) 1669.

8. H. Nishino and E. Sezgin, Nucl. Phys. B278 (1986) 353-379.

9. U. Theis and P. van Nieuwenhuizen, Class. Quant. Grav. 18 (2001) 54695486.

10. S. Weinberg, Gravitation and Cosmology, John Wiley 1972, Section 12.5.

11. L. Baulieu, M. Bellon and A. Tanzini, Eight-Dimensional Topological Gravity and its Correspondence with Supergravity, PAR-LPTHE 02-06, hepth/0207020.

12. B. Spence, Topological Born-Infeld Actions and D-Branes, hep-th/9907053.

13. R. Gopakumar and C. Vafa, Adv. Theor. Math. Phys., 3 (1999) 1415, hepth/9811131; J. Labastida and M. Mariño, Journal of Knot Theory and Its Ramifications, 112 (2002) 173-197, math.QA/0104180.

14. P. de Medeiros, J. Figueroa-O'Farrill, C. Hull and B. Spence, Conformal topological Yang-Mills theory and de Sitter holography, JHEP 08 (2002) 055, hep-th/0111190. 